\title{
Peptidi natriuretici e broncopneumopatia cronica ostruttiva nel Dipartimento di Emergenza
}

\section{$B$-type natriuretic peptide and COPD in the Emergency Department}

\section{Tiziano Minora *, Pietro Marino}

\author{
SC Medicina d'Urgenza, Azienda Ospedaliera "Fatebenefratelli e Oftalmico", Milano
}

Ricevuto il 28 novembre 2009; accettato il 19 giugno 2010

disponibile online il 16 settembre 2010

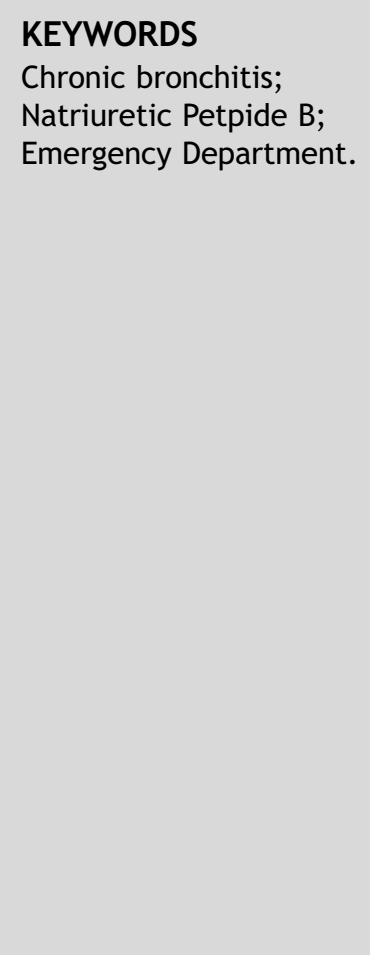

\begin{abstract}
Summary
Introduction: The aim of this study was to evaluate, in patients with chronic obstructive pulmonary disease (COPD) and arrived to the emergency department (ED) with dyspnea / hypoxia, the existence of possible correlations between blood levels of B-type natriuretic peptide (NT-proBNP) and presence of heart failure [HF]), mortality, outcome of the ED visit (discharge, ospitalization, death), and length of post-ED hospital stays.

Materials and methods: Clinical history, medical examination and blood levels of NT-proBNP were perfomed in all patients selected to our emergency department with the above symptoms between December 2006 and December 2008. Emergency department and hospital charts for these patients were later retrospectively reviewed to identify patients diagnosed with COPD. Results: A total of 546 patients (mean age 77 years, 52\% women) had final diagnoses of COPD (with or without HF). Eight died in the ED, 104 were discharged after the ED visit, and 424 were hospitalized. Hospitalizations were longer for patients with COPD alone ( 21 days vs. 13 days for COPD + HF). Mortality among hospitalized patients was $9.7 \%$. Seventy-six percent of the patients had elevated blood levels of NT-proBNP $(>450 \mathrm{ng} / \mathrm{L})$ in the ED. Most of these patients $(54 \%)$ had COPD and HF; the others $(22 \%)$ had COPD alone $(p<0.01)$. Low levels of NT-proBNP $(<450 \mathrm{ng} / \mathrm{L})$ were more frequent in patients with simple COPD who were discharged after the ED visit (89/ $546)$. In this group, $25 / 89$ returned to the ED after more than 60 days; $17 \%$ had simple COPD, and $10 / 25$ were hospitalized. Elevated blood NT-proBNP levels were significantly associated with mortality $(\mathrm{p}<0.001)$.

Discussion: The findings of the present study indicate that HF is the main cause of dyspnea and hypoxia among patients with COPD who present to the ED with these symptoms. High blood levels of NT-proBNP on ED admission can alert ED physicians to the presence of HF, allowing them to prescribe appropriate treatment and make more informed decisions regarding the need for hospitalization. Low levels of NT-proBNP appear to be associated with simple COPD and low-risk
\end{abstract}

\footnotetext{
* Corrispondenza: SC Medicina d'Urgenza, Azienda Ospedaliera "Fatebenefratelli e Oftalmico", c.so di Porta Nuova 23 - 20121 Milano. E-mail: timinora@tin.it (T. Minora).
} 
that may be manageable on an outpatient basis. Measurement of NT-proBNP levels in dyspneic patients presenting to the ER is helpful for making a correct diagnosis and identifying appropriate management strategies.

(c) 2010 Elsevier Srl. All rights reserved.

\section{Introduzione}

La broncopneumopatia cronica ostruttiva (BPCO) è una delle malattie a maggiore diffusione nel mondo. Negli Stati Uniti è stato stimato che ne siano affetti oltre 16 milioni di adulti, mentre l'incidenza complessiva in Europa e Nord America è di oltre 24 milioni [1-4]. Negli Stati Uniti è stato inoltre calcolato che per malattie polmonari, come BPCO e asma bronchiale, siano annualmente eseguite oltre 34 milioni di visite mediche con un costo superiore a 20 miliardi di dollari [2-4]. Secondo l'Organizzazione Mondiale della Sanità, entro il 2020 la BPCO sarà la terza causa di decesso nel mondo, con una stima di circa 20-30 milioni di vittime [1-4].

In Italia è la quinta malattia cronica per importanza, colpisce oltre 2,6 milioni di persone con una prevalenza del 4,5\% e una mortalità di circa 18.000 pazienti/anno. Il costo medio/paziente/anno è di circa 5.000-6.000 euro, prevalentemente $(70 \%)$ indotti dai costi di degenza [5-7].

Studi clinici hanno definito che morbilità e mortalità della BPCO siano correlate alla presenza di altre malattie, quali: coronaropatia, ipertensione arteriosa, diabete mellito e soprattutto scompenso cardiaco (SC) $[6,8-10]$. Non essendo presenti segni e sintomi specifici, la coesistenza di BPCO e SC costituisce ancora oggi, specie nelle fasi iniziali, una delle più complesse diagnosi differenziali nei pazienti afferiti in Pronto Soccorso [2,4,11-14]. Questa problematica condiziona sia la scelta di un approccio terapeutico maggiormente intensivo e invasivo sia le modalità di dimissione dal Pronto Soccorso [11,15-24].

Il Brain Natriuretic Peptide (BNP) è un polipeptide di 32 aminoacidi secreto principalmente a livello dei ventricoli del cuore in risposta a un "cardiac stretch" indotto da un sovraccarico pressorio o volumetrico [25-31]. I livelli ematici del peptide sono inoltre variabili, nei sessi, con l'età, nell'ipertensione polmonare, in risposta all'ipossia, a seguito di secrezione di citochine proinfiammatorie e per attivazione del sistema adrenergico [4,28-31]. Recenti studi hanno osservato che incrementi del BNP si riscontrano anche nella sola miocardiopatia destra [26,27]. Vari autori hanno inoltre dimostrato per il BNP sia un ruolo predittivo nella diagnosi di SC sia un ruolo prognostico e di guida nella terapia dello SC $[15,20,32-36]$.

\section{Materiali e metodi}

Lo studio è stato condotto presso il Dipartimento Emergenza Accettazione (DEA) dell'Azienda Ospedaliera "Fatebenefratelli e Oftalmico" di Milano, nei pazienti che si presentavano con dispnea e ipossia (saturazione $<90 \%$ ), allo scopo di valutare la predittività offerta dai diversi livelli di NT-proBNP nel discriminare, nel DEA e/o dopo degenza, la presenza di $\mathrm{BPCO}$ e/o SC, la possibilità di dimissione già in Pronto Soccorso, la mortalità e il tempo di degenza in ospedale.
La casistica raccolta da dicembre 2006 a dicembre 2008 comprende 546 pazienti con diagnosi di BPCO basata sulla storia clinica riferita, sulla documentazione presentata all'arrivo in Pronto Soccorso o rintracciabile attraverso l'archivio informatico disponibile presso la nostra Azienda e, nei pazienti ricoverati, in base all'analisi delle cartelle. Sono stati esclusi pazienti con età $<18$ anni, anamnesi di recente trauma toraco-addominale, gravide o non rintracciabili per il follow-up.

Al momento dell'arrivo in Pronto Soccorso i pazienti sono stati sottoposti, dai medici internisti della Medicina d'Urgenza o dai cardiologi, a valutazione clinica con raccolta dell'anamnesi clinica e farmacologica, visita medica, rilevazione dell'ossimetria capillare, esecuzione del dosaggio di NT-proBNP associato a esami ematochimici ed eventuali esami strumentali. Il medico prescriveva le procedure diagnostiche e terapeutiche opportune e, infine, stabiliva la modalità di dimissione dal Pronto Soccorso integrando la valutazione clinica con le informazioni ricavate dai livelli di NT-proBNP.

Nei pazienti ricoverati il follow-up è stato realizzato mediante l'analisi delle cartelle cliniche con verifica degli accertamenti eseguiti durante la degenza come spirometria, emogasanalisi ed eventuale broncoscopia e TC polmonare per la diagnosi BPCO ed ecocolordoppler cardiaco per la diagnosi di insufficienza cardiaca. Nei pazienti dimessi già in Pronto Soccorso, sono stati eseguiti un controllo telefonico (entro 60 giorni) e/o la verifica di un nuovo accesso in Pronto Soccorso mirati alla rivalutazione della correttezza della diagnosi proposta e delle modalità di dimissione.

Nei pazienti ricoverati sono stati raccolti, oltre alle diagnosi di dimissione, anche i tempi di degenza e le modalità di dimissione dal reparto; per i pazienti rinviati al domicilio, invece, sono stati raccolti i dati relativi all'incidenza e all'intervallo da un nuovo accesso in Pronto Soccorso.

\section{Dosaggio del peptide natriuretico}

Il dosaggio del peptide è stato eseguito attraverso la ricerca della frazione NT-proBNP, frammento inattivo del BNP ma con emivita più lunga, nel plasma eparinato con metodica Immuno Assay in elettrochemiluminescenza su immunoanalizzatori Stratus CS Acute Care (Dade Behring Vertriebs $\mathrm{GmbH}$, Germania) e con tempo di refertazione $<1$ ora, tale da essere disponibile per la valutazione clinica eseguita in Pronto Soccorso. È stato inoltre stabilito che $\mathrm{i}$ valori di dosaggio massimo non sarebbero stati $>40.000 \mathrm{ng} / \mathrm{L}$, poiché clinicamente già significativamente indicativi. Nella valutazione clinica sono stati proposti 2 cut-off: il primo, $<125 \mathrm{ng} /$ L, permetteva di escludere la presenza di SC "rule out"; il secondo, > $450 \mathrm{ng} / \mathrm{L}$, era indicativo della coesistenza di miocardiopatia acuta e/o cronica "rule in". La successiva analisi dei dati è stata effettuata in base al livello di "rule in" > 450 ng/L. 


\section{Analisi statistica}

L'analisi statistica è stata realizzata usando i software SPSS per Windows (versione 11) e Microsoft Office Excel 2007 per Windows. I valori sono stati espressi come medie (intervallo di confidenza al 95\%) e la significatività statistica è stata calcolata con livello $<0,05$. I confronti sono stati realizzati mediante l'esecuzione del $t$ test e del test del Chi quadro.

Lo studio è stato svolto a seguito di un protocollo di implementazione multidisciplinare che ha coinvolto Medicina d'Urgenza, UTIC-Cardiologia, SMeL (Medicina di Laboratorio) e Direzione Sanitaria Aziendale.

\section{Risultati}

Dalla casistica raccolta da dicembre 2006 a dicembre 2008, sono stati selezionati 546 pazienti con diagnosi di BPCO dopo follow-up. L'età media dei pazienti con BPCO era di 78,1 anni, con una prevalenza di pazienti di ultrasettantenni (80\%) e delle donne (53\%) (tabella 1).

La contemporanea presenza di più patologie è una caratteristica rilevante del campione in esame (tabella 2). Anamnesticamente la BPCO era già presente nell' $84 \%$ dei pazienti, l'ipertensione arteriosa nel $42 \%$, lo SC nel 34\%, la coronaropatia nel 26\%, il diabete mellito nel 22\% (tabella 1). Una sola comorbilità, associata alla BPCO, era presente nel $9 \%$ dei pazienti, 2 nel $64 \%$, mentre l' $8 \%$ presentava 3 o più malattie.

Una maggiore incidenza di comorbilità è presente nei pazienti con coesistenza di BPCO e SC (tabella 1). La diagnosi di BPCO è passata dal $27,2 \%$ anamestico al $32,3 \%$ dopo followup ( fig. 1), con un incremento di 87 nuovi casi determinato prevalentemente dalla comparsa di sola BPCO in 34/87 e di $\mathrm{BPCO}$ e SC in 53/87. La coesistenza di BPCO e SC è risultata aumentata da 161/459 a 324/546 prevalentemente per diagnosi misconosciuta di SC (110/163; 67\%), mentre le nuove diagnosi di BPCO e BPCO con SC sono state rispettivamente il $17 \%$ e il $16 \%$.

Tabella 1 Caratteristiche anamnestiche dei pazienti con BPCO.

\begin{tabular}{|c|c|c|c|}
\hline CASISITICA & $\begin{array}{c}\text { BPCO } \\
N=222\end{array}$ & $\begin{array}{c}\mathrm{BPCO}+\mathrm{SC} \\
\mathrm{N}=324\end{array}$ & $\begin{array}{l}\text { BPCO totali } \\
\mathrm{N}=546\end{array}$ \\
\hline Età (anni) & Media: 77,6 (32-100) & Media: 79,7 (48-101) & Media: 78,1 (31-101) \\
\hline Sesso & $\begin{array}{l}M: 108(49 \%) \\
F: 114(51 \%)\end{array}$ & $\begin{array}{l}M: 150(38 \%) \\
F: 174(62 \%)\end{array}$ & $\begin{array}{l}M: 258(47 \%) \\
F: 288(53 \%)\end{array}$ \\
\hline DATI ANAMNESTICI & $\begin{array}{c}\text { N. casi } \\
0\end{array}$ & $\begin{array}{c}\text { N. casi } \\
188(58 \%)\end{array}$ & $\begin{array}{c}\text { N. casi } \\
188(34 \%)\end{array}$ \\
\hline BPCO & $188(85 \%)$ & $271(84 \%)$ & $459(84 \%)$ \\
\hline $\mathrm{SC}+\mathrm{BPCO}$ & 0 & $161(50 \%)$ & $161(29 \%)$ \\
\hline Ipertensione arteriosa & $93(42 \%)$ & $135(42 \%)$ & $228(42 \%)$ \\
\hline Diabete mellito & $29(13 \%)$ & $193(60 \%)$ & $122(22 \%)$ \\
\hline Coronaropatia & $41(18 \%)$ & $101(31 \%)$ & $142(26 \%)$ \\
\hline Infarto miocardico (anamnesi) & 24 & 69 & 93 \\
\hline Cardiopatia Ischemica Cronica & 17 & 32 & 49 \\
\hline Aritmie totali & $38(17 \%)$ & $135(42 \%)$ & $173(32 \%)$ \\
\hline Fibrillazione atriale parossistica & 4 & 33 & 47 \\
\hline Fibrillazione atriale cronica & 6 & 45 & 51 \\
\hline Tachicardia sopraventricolare parossistica & 4 & 6 & 10 \\
\hline Pacemaker (presenza) & 16 & 51 & 67 \\
\hline Insufficienza renale cronica & $14(6 \%)$ & $59(18 \%)$ & $73(13 \%)$ \\
\hline Anemia & $10(5 \%)$ & $25(8 \%)$ & $35(6 \%)$ \\
\hline Dislipidemia & $22(10 \%)$ & $44(14 \%)$ & $66(12 \%)$ \\
\hline Neoplasia & $28(13 \%)$ & $31(10 \%)$ & $59(11 \%)$ \\
\hline TERAPIE & N. casi & N. casi & N. casi \\
\hline Farmaci bronchodilatatori & $95(43 \%)$ & $113(35 \%)$ & $208(38 \%)$ \\
\hline Steroidi per os/intramuscolo & $33(15 \%)$ & $38(12 \%)$ & $71(8 \%)$ \\
\hline Teofillina & $11(5 \%)$ & $23(7 \%)$ & 34 (9\%) \\
\hline ACE-inibitori/Sartanico & 75 (32\%) & $136(42 \%)$ & 211 \\
\hline Diuretici & 72 (32\%) & 235 (76\%) & 307 (31\%) \\
\hline Beta-bloccanti & $20(9 \%)$ & $66(21 \%)$ & $86(18 \%)$ \\
\hline
\end{tabular}


Tabella 2 Pazienti con BPCO e con BPCO e altre malattie.

\begin{tabular}{|c|c|c|c|c|}
\hline Parametro & $\begin{array}{l}\text { BPCO totali } \\
\qquad N=546\end{array}$ & $\begin{array}{c}\text { BPCO } \\
N=103\end{array}$ & $\begin{array}{c}\text { BPCO + altre malattie } \\
\qquad N=443\end{array}$ & Statistica \\
\hline Rapporto F/M & 1,01 & 1,1 & 1,2 & \\
\hline Età (anni): media & 78,1 & 75,4 & 78,7 & $\mathrm{p}<0,001$ \\
\hline BNP: media & 4958 & 3422 & 5236 & $\mathrm{p}<0,001$ \\
\hline BNP $<450$ & 122 & 39 & 94 & $p<0,001$ \\
\hline $\mathrm{BNP}>450$ & 414 & 64 & 349 & \\
\hline \multirow[t]{2}{*}{ Outcome Pronto Soccorso } & Dimissione:104 & Dimissione: 18 & Dimissione: 86 & \\
\hline & Ricovero: 424 & Ricovero: 85 & Ricovero: 349 & \\
\hline Decesso in Pronto Soccorso & 8 & 0 & 8 & \\
\hline Modalità dimissione/ NT-proBNP & & & & $\mathrm{p}<0,001$ \\
\hline
\end{tabular}

Il valore medio di NT-proBNP è stato globalmente di 4.958 $\mathrm{ng} / \mathrm{L}$ con una significativa prevalenza $(\mathrm{p}<0,001)$ nei pazienti con contemporanea presenza di SC rispetto a quelli con sola BPCO ( fig. 2). Nella nostra casistica prevalgono i pazienti con elevati livelli del peptide (414/546; 76\%), mentre è modesta la quota (7\%) con bassi livelli di NT-proBNP ( fig. 3). Valori elevati del peptide prevalgono nei pazienti con contemporanea presenza di BPCO e SC rispetto a quelli con sola BPCO $(90,7 \%$ vs $54 \%)$, mentre bassi livelli del peptide sono più diffusi nei pazienti con sola BPCO (77\%) ( fig. 3).

Le cause di accesso in Pronto Soccorso sono state la presenza di SC in 193/546, di BPCO in 174/546, di altre malattie (scompenso cardiaco acuto, embolia polmonare, sepsi ecc.) in 179/546.

Nel gruppo di pazienti con BPCO e SC ( $\mathrm{N}=324)$ la causa di arrivo in Pronto Soccorso è stata lo SC nel $60 \%$, la BPCO riacutizzata e/o la polmonite nel $26 \%$, altre malattie nel $14 \%$, mentre nei pazienti senza SC ( $N=222)$ solo il $47 \%$ ha manifestato una BPCO riacutizzata.

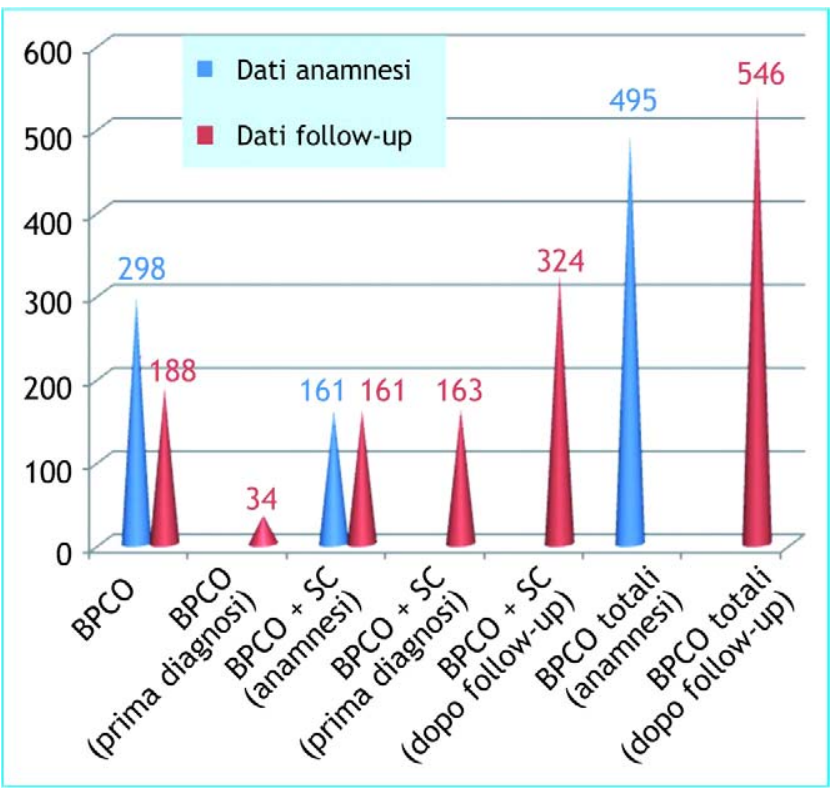

Figura 1 Incidenza di BPCO e SC anamnestica e dopo follow-up.
Nei 169 pazienti con "prima diagnosi" di BPCO e SC, elevati livelli di NT-proBNP erano presenti nell'88\%; l'81\% è stato ricoverato. Tra i pazienti con nuova diagnosi di BPCO senza SC $(\mathrm{N}=34)$ solo il $26 \%$ presentava bassi livelli del peptide.

L'analisi della modalità di dimissione dal Pronto Soccorso ha evidenziato uno stretto rapporto fra elevati livelli di NTproBNP, presenza contemporanea di SC e ricovero (92\%) ( $p<0,01$ ), mentre bassi livelli di NT-proBNP non sono correlati con la dimissione dal Pronto Soccorso (36\%) (fig. 4). Solamente $89 / 546$ pazienti sono stati dimessi già in Pronto Soccorso e solo $25 / 89$ si sono ripresentati in ospedale, in media dopo oltre 60 giorni. Questi erano affetti da BPCO senza SC (17\%) e 10/25 sono stati ricoverati prevalentemente dopo 30-60 giorni.

I pazienti con o senza SC hanno presentato una mortalità globale del $9,7 \%$ senza differenza di incidenza fra quella osservata in Pronto Soccorso $(1,2 \%$ vs $1,8 \%)$ e quella durante ricovero $(7,7 \%$ vs $7,2 \%)$.

La degenza media è risulta superiore nei pazienti con BPCO rispetto a quelli con BPCO e SC (21,3 vs 13,8 giorni) ed elevati valori di NT-proBNP sono stati prevalenti $(p=0,01)$ nei pazienti con prognosi infausta $(49 / 51)$.

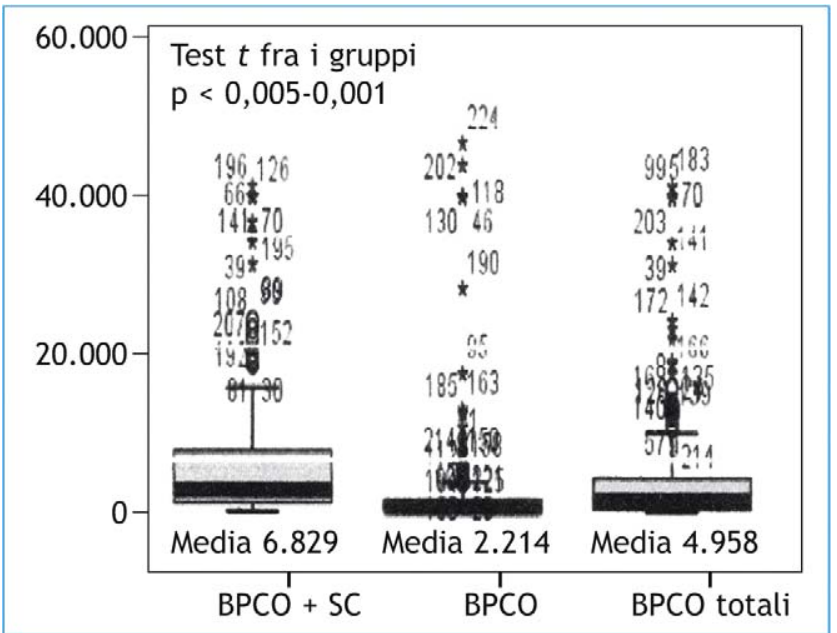

Figura 2 Livelli di NT-proBNP in pazienti con BPCO. 


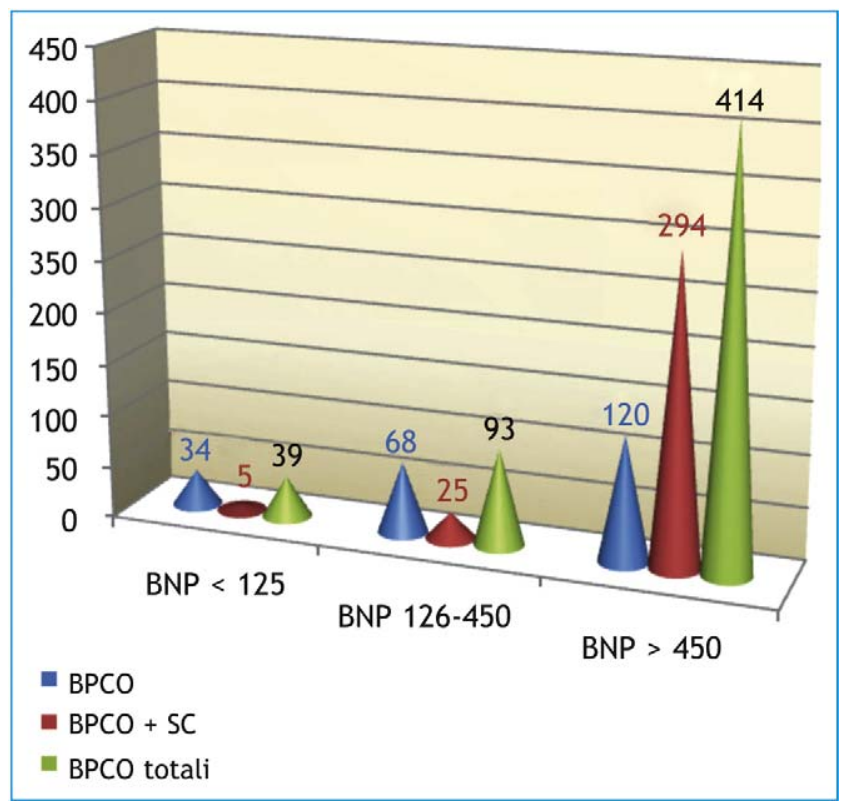

Figura 3 Rapporto fra livelli di NT-proBNP e pazienti con BPCO.

\section{Discussione}

La nostra casistica, anche se riferita a un campione selettivo, rispecchia le caratteristiche epidemiologiche dei pazienti affetti da BPCO: prevalenza di donne (53\%), pazienti di età $>70$ anni (80\%) e affetti da plurime patologie (81\%) (tabella 1) [4-6,18]. Dopo follow-up ( fig. 1) l'incidenza totale della BPCO è aumentata del $6 \%$ rispetto al dato anamnestico, con una diminuzione dei pazienti con sola BPCO, sebbene siano stati diagnosticati 34 nuovi casi, e una prevalenza di pazienti con contemporanea presenza di SC per osservazione di 163 nuove diagnosi.

La presenza di pazienti con BPCO e SC è passata dal $29 \%$ anamnestico al 59\%. Analogamente ad altri studi $[8,15,31]$ la causa principale della dispnea è stata l'insufficienza cardiaca, di nuova diagnosi o recidivante (35\%), mentre riacutizzazioni della BPCO sono state osservate solo nel $31 \%$.

La rilevante presenza di comorbilità anamnestiche e/o dopo follow-up (tabella 1), come sottolineato anche da altri autori $[16,20,33]$, può rendere difficile una corretta valutazione in Pronto Soccorso del rapporto fra livelli ematici di NTproBNP e predittività di miocardiopatia. Fra i pazienti indagati prevale l'osservazione di elevati valori di NT-proBNP (76\%), condizione questa indotta sia, secondo Ishii e Stolz $[8,26,38]$, dalla presenza di ipertensione polmonare sia dalla contemporanea presenza di malattie cardiache $(63 \%)$ e/o di altra eziologia che possono indirettamente provocare miocardiopatie secondarie $[1,3,20,26,33]$.

Anche nella nostra casistica, similmente a quanto rilevato da altri autori, sono emerse sia una buona correlazione fra bassi livelli di NT-proBNP, assenza di SC e/o patologia ad alto rischio sia una predittività positiva di alti valori del peptide come indicatore di SC (91\%) [2,15-18,25,32,35-38]. Inoltre, analizzando il campione queste incidenze non subiscono significative variazioni statistiche e soprattutto cliniche se si esaminano valori soglia differenti (> $300 \mathrm{ng} / \mathrm{L}$ o $900 \mathrm{ng} / \mathrm{L}$ ) come proposti da vari autori $[15,19,20]$. Dopo follow-up è apparso rilevante l'incremento dei pazienti con diagnosi di SC (193/324), dapprima misconosciuta in 136/193 (70\%), che ha determinato un'inversione nell'incidenza dei pazienti con e senza SC (fig. 1). Tali variazioni sono prevalentemente associate alla presenza di livelli elevati di NT-proBNP e potrebbero essere espressione di un'iniziale miocardiopatia misconosciuta o di uno stadio preclinico dello SC [38-48].

Nella valutazione dell'utilità del dosaggio del NT-proBNP come marcatore da affiancare alla diagnostica clinica tradizionale per la determinazione della necessità del ricovero ( fig. 1), come evidenziato da svariati autori tra cui Mueller et al. e Moe et al. [14,15,18-20,24,29,42-45], emerge un positivo riscontro poiché nella nostra casistica l' $86 \%$ dei ricoverati aveva alti livelli del peptide e il $17 \%$ dei pazienti con bassi valori del peptide è stato ricoverato, spesso per malattie correlate alle comorbilità presenti. Nel gruppo di pazienti rinviati al domicilio (104/546), il 45\% presenta bassi livelli di NT-proBNP e patologie a basso rischio, solo il $25 \%$ si è

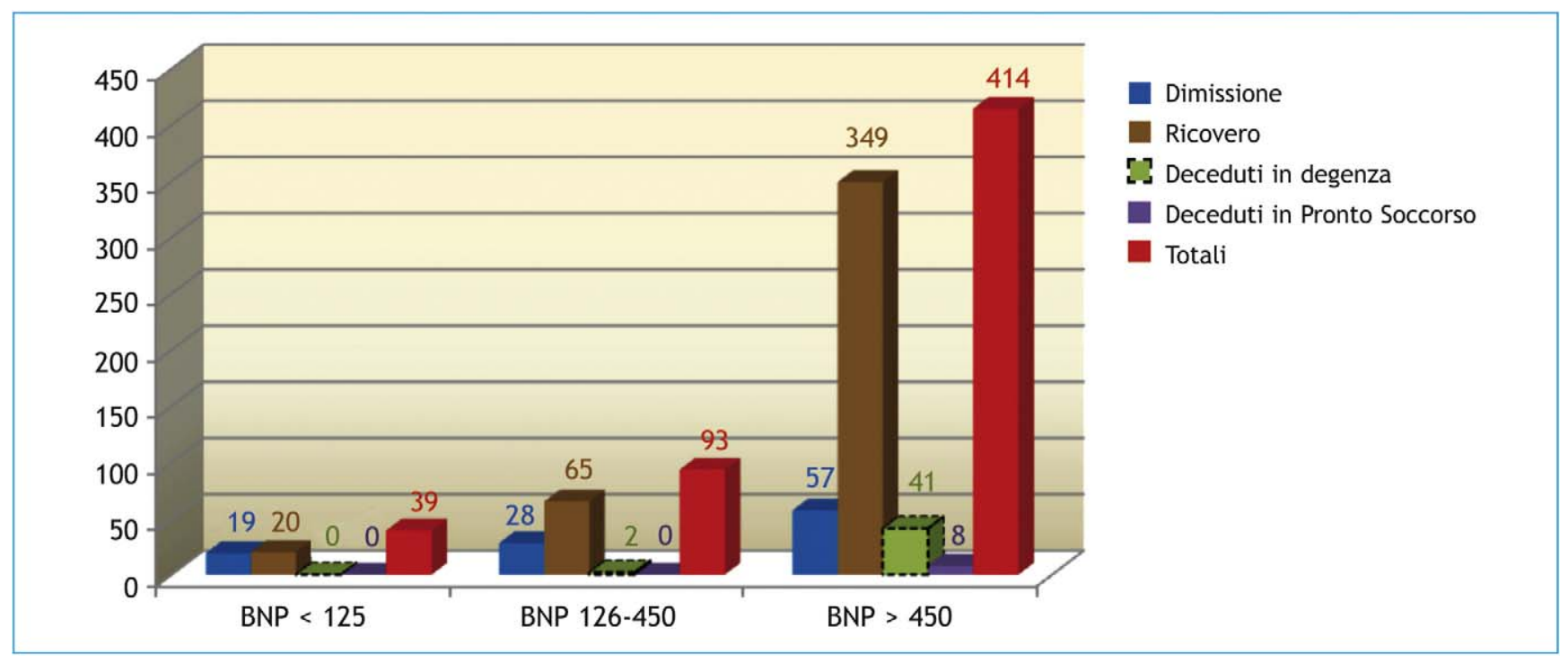

Figura 4 Rapporto fra livelli NT-proBNP e decisione finale in Pronto Soccorso. 
ripresentato in Pronto Soccorso e l'1,8\% è stato ricoverato dopo oltre 60 giorni dal precedente arrivo in ospedale come evidenziato anche da altri AA [47-49].

La mortalità osservata è stata dell'8\%, e in tutti i casi si è trattato di pazienti affetti da BPCO e SC, analogamente a quanto osservato da Mueller et al., ma inferiore a quella rilevata nello studio EPIDASA e in altri lavori, in cui era compresa fra il 16 e il $21 \%[8,14,16,27,32,39,45,46]$.

Nell'analisi della prognosi a breve termine si conferma anche il ruolo predittivo dei livelli ematici di NT-proBNP: nella nostra casistica il 98\% dei pazienti deceduti in Pronto Soccorso o durante la degenza presentava livelli ematici > $4.000 \mathrm{ng} / \mathrm{L}$.

La differenza nei tempi di degenza non sembra attribuibile ai soli criteri di gravità della malattia, ma anche alla scelta di diverse modalità di dimissione.

Come già sottolineato dalla task force della European Society of Cardiology, esistono sufficienti evidenze cliniche $[24,34,35,49]$ riguardo al fatto che la valutazione dei livelli dei peptidi natriuretici BNP o NT-proBNP, anche se non deve essere considerata criterio sufficiente per "rule in" per diagnosi di insufficienza cardiaca, può offrire, nei pazienti con dispnea, un utile supporto al medico del Pronto Soccorso, specie in assenza di indagini strumentali o storia clinica, per porre la diagnosi di SC o di patologie ad alto rischio (infarto miocardico, embolia polmonare, sepsi) e conseguentemente anche nelle scelte terapeutiche, con miglioramento della prognosi e possibile riduzione dei costi.

\section{Conflitto di interesse}

Gli autori dichiarano di essere esenti da conflitto di interessi.

\section{Bibliografia}

[1] Pauwels RA, Rabe KF. Burden and clinical features of chronic obstructive pulmonary disease (COPD). Lancet 2004;364: 613-20.

[2] American Thoracic Society. Dyspnea. Mechanisms, assessment, and management: a consensus statement. Am J Respir Crit Care Med 1999;15:321-40.

[3] American Thoracic Society. Standards for the diagnosis and care of patients with chronic obstructive pulmonary disease. Am J Respir Crit Care Med 1995;152:S77-121.

[4] Higgins M. Epidemiology of obstructive pulmonary disease. In: Cassaburi R, Petty TL, editors. Principles and Practice of Pulmonary Rehabilitation. Philadelphia, PA: WB Sauders; 1993.

[5] Scognamiglio A, Matteelli G, Pistelli F, et al. Epidemiology of chronic obstructive pulmonary disease. Ann Ist Super Sanità 2003;39:467-84.

[6] Linee Guida GOLD. Progetto mondiale bronco pneumopatia cronica ostruttiva (BPCO). www.goldcopd.it/

[7] Dal Negro RW, Bonadiman L, Turati C, et al. Clinical and pharmacoeconomic profile of COPD patients with FEV1 50 $60 \%$ predicted: pilot study on the impact of the extended indication of ICS/LABA. Ther Adv Respir Dis 2009;3:51-8.

[8] Stolz D, Breidthardt T, Christ-Crain M, et al. Use of B-type natriuretic peptide in the risk stratification of acute exacerbations of COPD. Chest 2008;133:1088-194.

[9] Soler-Cataluña JJ, Martìnez-Garcìa MA, Romàn Sànchez P, et al. Severe acute exacerbations and mortality in patients with chronic obstructive pulmonary disease. Thorax 2005;60: 925-31.
[10] Antonelli IR, Fuso L, De Rosa M, et al. Co-morbidity contributes to predictmortality of patients with chronic obstructive pulmonary disease. Eur Respir J 1997;10:2794-800.

[11] Cabanes L, Richaud-Thiriez B, Fulla Y, et al. Brain natriuretic peptide blood levels in the differential diagnosis of dyspnea. Chest 2001;120:2047-50.

[12] Christ M, Thuerlimann A, Laule K, et al. Long-term prognostic value of $\mathrm{B}$-type natriuretic peptide in cardiac and non-cardiac causes of acute dyspnoea. Eur J Clin Invest 2007;37:834-41.

[13] Abroug F, Ouanes-Besbes L. Detection of acute heart failure in chronic obstructive pulmonary disease patients: role of B-type natriuretic peptide. Curr Opin Crit Care 2008;14:340-7.

[14] Ray P, Birolleau S, Lefort Y, et al. Acute respiratory failure in the elderly: etiology, emergency diagnosis and prognosis. Crit Care 2006;10:R82.

[15] Januzzi Jr JL, Camargo CA, Anwaruddin S, et al. The N-terminal Pro-BNP investigation of dyspnea in the emergency department (PRIDE) study. Am J Cardiol 2005;95:948-54.

[16] Mueller C, Laule-Kilian K, Frana B, et al. Use of B-type natriuretic peptide in the management of acute dyspnea in patients with pulmonary disease. Am Heart J 2006;151:471-7.

[17] Cheng V, Kazanagra R, Garcia A, et al. A rapid bedside test for B-type peptide predicts treatment outcomes in patients admitted for decompensated heart failure: a pilot study. J Am Coll Cardiol 2001;37:386-91.

[18] Mueller C, Scholer A, Laule-Kilian K, et al. Use of B-type natriuretic peptide in the evaluation and management of acute dyspnea. N Engl J Med 2004;350:647-54.

[19] Harrison A, Morrison LK, Krishnaswamy P, et al. B-type natriuretic peptide predicts future cardiac events in patients presenting to the emergency department with dyspnea. Ann Emerg Med 2002;39:131-8.

[20] Maisel AS, Krishnaswamy P, Nowak RM. et al., Breathing Not Properly Multinational Study Investigators. Rapid measurement of B-type natriuretic peptide in the emergency diagnosis of heart failure. N Engl J Med 2002;347:161-7.

[21] Baggish AL, Cameron R, Anwaruddin S, et al. A clinical and biochemical critical pathway for the evaluation of patients with suspected acute congestive heart failure: The ProBNP Investigation of Dyspnea in the Emergency Department (PRIDE) algorithm. Crit Pathw Cardiol 2004;3:171-6.

[22] Maisel AS, McCord J, Nowak RM, et al., Breathing Not Properly Multinational Study Investigators. Bedside B-Type natriuretic peptide in the emergency diagnosis of heart failure with reduced or preserved ejection fraction. Results from the Breathing Not Properly Multinational Study. J Am Coll Cardiol 2003;41:2010-7.

[23] Maisel A, Hollander JE, Guss D, et al., Rapid Emergency Department Heart Failure Outpatient Trial investigators. Primary results of the Rapid Emergency Department Heart Failure Outpatient Trial (REDHOT). A multicenter study of B-type natriuretic peptide levels emergency department decision making and outcomes in patients presenting with shortness of breath. J Am Coll Cardiol 2004;44:1328-33.

[24] Siebert U, Januzzi JL, Beinfeld MT, et al. Cost effectiveness of using NT-proBNP to guide the diagnostic assessment and management of dyspneic patients in the emergency department. Presented at 2004 American Heart Association Meeting (New Orleans, LA).

[25] Mueller C, Breidthardt T, Laule-Kilian K, Christ M, Perruchoud AP. The integration of BNPand NT-proBNP into clinical medicine. Swiss Med Wkly 2007;137:4-12.

[26] Ishii J, Nomura M, Ito $M$, et al. Plasma concentration of brain natriuretic peptide as a biochemical marker for the evaluation of right ventricular overload and mortality in chronic respiratory disease. Clin Chim Acta 2000;301:19-30.

[27] Leuchte HH, Holzapfel M, Baumgartner RA, et al. Clinical significance of brain natriuretic peptide in primary pulmonary hypertension. J Am Coll Cardiol 2004;43:764-70. 
[28] Nagaya N, Nishikimi T, Uematsu M, et al. Plasma brain natriuretic peptide as a prognostic indicator in patients with primary pulmonary hypertension. Circulation 2000;102:865-70.

[29] Clerico A, Del Ry S, Maffei S, Prontera C, Emdin M, Giannessi D. The circulating levels of cardiac natriuretic hormones in healthy adults: effects of age and sex. Clin Chem Lab Med 2002;40:371-7.

[30] Silver MA. The natriuretic peptide system: kidney and cardiovascular effects. Curr Opin Nephrol Hypertens 2006;15:14-21.

[31] Ando T, Ogawa K, Yamaki K, Hara M, Takagi K. Plasma concentrations of atrial, brain, and $\mathrm{C}$-type natriuretic peptides and endothelin-1 in patients with chronic respiratory diseases. Chest 1996;110:462-8.

[32] Gardner RS, Ozalp F, Murday AJ, Robb SD, McDonagh TA. $\mathrm{N}$-terminal pro-brain natriuretic peptide. A new gold standard in predicting mortality in patients with advanced heart failure. Eur Heart J 2003;24:1735-43.

[33] Tung RH, Garcia C, Morss AM, et al. Utility of B-type natriuretic peptide for the evaluation of intensive care unit shock. Crit Care Med 2004;32:1643-7.

[34] Swedberg K, Cleland J, Dargie H, et al. Task Force for the Diagnosis and Treatment of Chronic Heart Failure of the European Society of Cardiology. Guidelines for the diagnosis and treatment of chronic heart failure: executive summary (update 2005): The Task Force for the Diagnosis and Treatment of Chronic Heart Failure of the European Society of Cardiology. Eur Heart J 2005;26:1115-40.

[35] Januzzi JL, Richards AM, editors. An international consensus statement regarding amino terminal pro-B-type natriuretic peptide testing: The International NT-proBNP Consensus Panel. Am J Cardiol 2008;101(3A).

[36] Berdagué P, Caffin PY, Barazer I, et al. Use of $\mathrm{N}$-terminal prohormone brain natriuretic peptide assay for etiologic diagnosis of acute dyspnea in elderly patients. Am Heart J 2006; 151:690-8.

[37] Verdiani V, Nozzoli C, Bacci F, et al. Pre-discharge B-type natriuretic peptide predicts early recurrence of decompensated heart failure in patients admitted to a general medical unit. Eur J Heart Fail 2005;7:566-71.

[38] Stolz D, Christ-Crain M, Morgenthaler NG, et al. Copeptin, Creactive protein, and procalcitonin as prognostic biomarkers in acute exacerbation of COPD. Chest 2007;131:1058-67.
[39] Fuso L, Incalzi RA, Pistelli R, et al. Predicting mortality of patients hospitalized for acutely exacerbated chronic obstructive pulmonary disease. Am J Med 1995;98:272-7.

[40] Suntharalingam J, Goldsmith K, Toshner M, et al. Role of NT-proBNP and 6MWD in chronic thromboembolic pulmonary hypertension. Respir Med 2007;101:2254-62.

[41] Bettencourt P, Azevedo A, Pimenta J, et al. N-terminalpro-brain natriuretic peptide predicts outcome after hospital discharge in heart failure patients. Circulation 2004;110: 2168-74.

[42] Bettencourt P. NT-proBNP and BNP: biomarkers for heart failure management. Eur J Heart Fail 2004;6:359-63.

[43] Seino Y, Ogawa A, Yamashita T, et al. Application of NT-proBNP and BNP measurements in cardiac care: a more discerning marker for the detection and evaluation of heart failure. Eur J Heart Fail 2004;6:295-300.

[44] Rihal CS, Davis KB, Kennedy JW, Gersh BJ. The utility of clinical, electrocardiographic, and roentgenographic variables in the prediction of left ventricular function. Am J Cardiol 1995;75: 220-3.

[45] Moe GW, Howlett J, Januzzi JL, Zowall H, Canadian Multicenter Improved Management of Patients With Congestive Heart Failure (IMPROVE-CHF) Study Investigators. N-terminal pro-B-type natriuretic peptide testing improves the management of patients with suspected acute heart failure: primary results of the Canadian prospective randomized multicenter IMPROVECHF study. Circulation 2007;115:3103-10.

[46] Mueller C, Laule-Kilian K, Scholer A, et al. Use of B-type natriuretic peptide for the management of women with dyspnea. Am J Cardiol 2004;94:1510-4.

[47] Logeart D, Thabut G, Jourdain P, et al. Predischarge B-type natriuretic peptide assay for identifying patients at high risk of re-admission after decompensated heart failure. J Am Coll Cardiol 2004;43:635-41.

[48] Doust JA, Pietrzak E, Dobson A, Glasziou P. How well does B-type natriuretic peptide predict death and cardiac events in patients with heart failure: systematic review. BMJ 2005;330: 625-539.

[49] Chenevier-Gobeaux C, Allo JC, Arthaud M, et al. N-Terminal pro B-type natriuretic peptide testing for short-term prognosis in breathless older adults. Am J Emerg Med 2008;26:555-60. 